\title{
Causal Logic of Minkowski Space
}

\author{
W. Cegła and A. Z. Jadczyk \\ Institute of Theoretical Physics, University of Wrocław, 50-205 Wroclaw, Poland
}

\begin{abstract}
It is shown that double-orthogonal sets (diamonds) in Minkowski space form an orthomodular complete lattice. Connection with empirical logic of Randall and Foulis is discussed.
\end{abstract}

\section{Introduction}

Relations of a causal type have been studied by various authors (see e.g. [1], and references there). In particular, Zeeman [2] has shown that every automorphism of the causal order structure of the Minkowski space is either Poincare transformation or dilation. This result has been generalized in [3-5], and finally Borchers and Hegerfeldt [6] have been able to show that preservation of light cones is sufficient to deduce the Zeeman's result (plus time inversion). On the other hand, the present authors [7], motivated by quantum mechanics and algebraic quantum field theory, started investigations of a causal-logic struture of space-time. In the Galilean case the most general covariant representation of the causal logic has been found to correspond to the quantum mechanics of an extended body. This was possible owing to the extremely simple structure of the Galilean logic, which happens to be nothing but a disjoint sum of Boolean logics. The causal structure of the Minkowski space is much less trivial. The present paper shows that the family of all doubleorthogonal sets is a complete orthomodular lattice (in fact, not a modular one). The full group of automorphisms of this logic consists of dilations and Poincaré transformations. In relativistic quantum mechanics one should have a covariant representation of this logic in the logic of projections of a Hilbert space. In quantum field theory projections are to be replaced by more general non-Abelian von Neumann algebras.

The methods and language we have found most appropriate are those of empirical logic of Randall and Foulis [8]. On the other hand, our example illustrates neatly all of the main concepts of Randall and Foulis approach. 


\section{Main Concepts of Empirical Logic}

The fundamental concept of Randall and Foulis approach is that of an operation. The set of all operations one is going to deal with is called a manual. If $\mathscr{A}$ is a manual, each operation $E \in \mathscr{A}$ is identified with the set of its possible outcomes $x \in E$. After appropriate identifications of outcomes of different operations one can assume that each $E \in \mathscr{A}$ is a subset of a set $M$ of all possible outcomes in the theory. Two different outcomes $x, y \in M$ are said to reject operationally each other if and only if there exists an operation $E$ such that both $x$ and $y$ are possible outcomes of $E$. In this case one writes $x \perp y$, and $x, y$ are said to be orthogonal. A subset $D$ of $M$ is called an orthogonal set provided every two different elements of $D$ are orthogonal. It is assumed that each $E \in \mathscr{A}$ is a maximal orthogonal set and conversely, every maximal orthogonal set corresponds to some (possibly idealized) operation. Under these assumptions the whole structure is completely specified by a symmetric, nonreflexive relation " $\perp$ " in $M$. An event is an $\perp$-set, and to each event $D$ there corresponds a proposition $\left(D^{\perp \perp}, D^{\perp}\right)$ (where $A^{\perp}=\{x \in M: x \perp y, y \in A\}$ ) confirmed when an operation $E$ gives an outcome $x \in D^{\perp \perp}$, and refuted if $x \in D^{\perp}$. It follows from the very defition that there always exists an operation (namely $E \supset D$ ) whose every outcome either confirms or refutes the proposition $p(D)=\left(D^{\perp \perp}, D^{\perp}\right)$. The set of all such propositions can be identified with the family $L(M, \perp)=\left\{D^{\perp \perp}: D-\perp\right.$-set $\}$. Equipped with the natural partial order " $\leqq$ " (inclusion) $L(M, \perp)$ is said to be the logic of $(M, \perp)$. If and only if $(M, \perp)$ satisfies the following Dacey condition (D), $L(M, \perp)$ is closed under orthocomplementation $A \mapsto A^{\perp}$, and is an orthomodular orthoposet ([9], see also [10]):

(D) $E \in \mathscr{A}, \quad x, y \in M, \quad E \subset\{x\}^{\perp} \cup\{y\}^{\perp} \Rightarrow x \perp y$.

In general, however, $L(M, \perp)$ need not be a lattice. A necessary and sufficient condition for $L(M, \perp)$ to be a complete orthomodular lattice is that the following condition (CD) holds [11]:

(CD) $A=A^{\perp \perp} C M$ and $D$-maximal $\perp$-set in $A \Rightarrow D^{\perp \perp}=A$.

Given this first order structure one can consider compound operations consisting of finite, ordered sequences of primitive outcomes. In the present paper we restrict ourselves to $L(M, \perp)$ only.

\section{The Causal Logic of Minkowski Space}

Before we go into details let us first give a brief motivation for the following construction. Let us take the point of view that in quantum mechanics position measurements are fundamental. In a non-relativistic case this is more or less obvious. In [12] it has been shown that momentum measurements can be reduced to those of position, and in $[13,14]$ scattering experiments have been described in terms of geometrical configurations of incoming and outgoing particles only. In a relativistic case the situation is not much different if one is going to pay the price of dealing with negative energy states. Once this viewpoint is accepted, it is natural to take as a fundamental operation an operation $E$ consisting of localization of a particle on a space- or light-like hypersurface $E(t=0$ hyperplane in the Galilean 
case). (In field theory one measures the field strength, and $E$ serves as a support for Cauchy data.) Clearly, having exactly one massive particle, two different outcomes $x, y \in E$ reject each other, and a proposition $p(D)$ can be realized experimentally by sensilizing a Geiger counter in $D$. Observe that a free particle trajectory intersects $D$ if and only if it intersects $D^{\perp \perp}$ (this is intuitively obvious and will be proved further). Thus $D_{1}$ and $D_{2}$ with $p\left(D_{1}\right)=p\left(D_{2}\right)$ can be really identified as experimental propositions. This motivates investigations of the logic $L(M, \perp), M$ being Minkowski space and " $\perp$ " meaning space- or light-like. ${ }^{1,2}$

We shall show now that equipped with such an orthogonality relation, Minkowski space is a complete Dacey space. Since the number of space dimensions plays no role in our argument (it can be infinite as well) we consider a general Minkowski space as defined below.

Let $V$ be a real vector space with a nondegenerate, bilinear symmetric form $(u, v)$, and let $C=\{v \in V:(v, v)<0\} . V$ is said to be a $\pi_{1}$-space if there exists $v \in C$ such that

$$
(v, u)^{2} \geqq(v, v)(u, u), \quad \forall u \in V .
$$

It is easy to see that $v \in C$ satisfies $\left(\pi_{1}\right)$ iff the scalar product restricted to $\{v\}^{\perp}$ is positive definite. Moreover, in a $\pi_{1}$-space, the $\left(\pi_{1}\right)$-condition is automatically satisfied for all $v \in C$. A particular example of a $\pi_{1}$-space is $V=R^{n}$, and $(u, v)$ $=\eta_{\mu \nu} u^{\mu} v^{\nu}, \eta=\operatorname{diag}(-1,+1, \ldots,+1)$. The set $C$ is nothing but the interior of the light cone.

Let us fix once for all a $\pi_{1}$-space $V$. Given $v \in C$, let for each $u \perp v,\|u\|^{2}=(u, u)$. Clearly, $\|\cdot\|$ is a norm on $\{v\}^{\perp}$.

An affine space $M$ over $V$ is called a Minkowski space over $V$. So, if $x, y \in M$, then $x-y \in V$, and each $u \in V$ defines a translation $x \mapsto x+u$ of $M$. We define orthogonality relation in $M$ by

$$
x \perp y \Leftrightarrow x \neq y \quad \text { and } \quad x-y \notin C, x, y \in M .
$$

The rest of this section is a study of " $\perp$ "-geometry of $M$ in order to show that $(M, \perp)$ is a complete $D$-space, i.e. to show that the condition (CD) of Section 2 holds in $M$.

Let us fix $a \in M$ and $v \in C$, and let $l$ be the straight line

$$
l=\{a+t v: t \in R\} .
$$

For each $x \in M$, we have a unique decomposition

$$
x=a+x^{0} v+\boldsymbol{x}, \quad \boldsymbol{x} \perp v, \quad x^{0} \in R .
$$

Let

$$
I(l ; x)=\{t: a-x+t v \notin C\},
$$

1 It may also be noted that a track of the particle in a Willson chamber can be regarded as an event for the second order logic. Here orthogonality means $x_{1} \ldots x_{n} \perp y_{1} \ldots y_{m}$ iff $x_{1} \ldots x_{k}=y_{1} \ldots y_{k}$ and $x_{k+1} \perp y_{k+1}$. Thus $x_{1} \ldots x_{n} \perp y_{1} \ldots y_{n}$ means that the two outcomes belong to two different branches of the Universe split after $k$ steps, according to Everett and Wheeler

2 One would also like to know if construction of $L(M, \perp)$ (modulo meager sets) is possible as a second order logic of some finite structure (compare [15]) 
so that $t \in I(l ; x)$ iff

$$
\left|t-x^{0}\right| \leqq\|\boldsymbol{x}\| /[-(v, v)]^{1 / 2}
$$

It follows, in particular, that $I(l ; x)$ is a non-empty, bounded closed interval.

Lemma 1. If $x-y \notin C$, then $I(l ; x) \cap I(l ; y) \neq \emptyset$.

Proof. If $v^{\prime}=s v, s \neq 0$, then $I\left(l^{\prime} ; x\right)=s^{-1} I(l ; x)$, and so, we can assume that $(v, v)=-1$. Therefore $t \in I(l ; x)$ iff $\left|t-x^{0}\right| \leqq\|\boldsymbol{x}\|$, and $x-y \notin C$ is equivalent to $\left|x^{0}-y^{0}\right| \leqq\|\boldsymbol{x}-\boldsymbol{y}\|$. We can thus assume that $\|\boldsymbol{x}-\boldsymbol{y}\| \neq 0$. With $t=s x^{0}+(1-s) y^{0}$, where $s=\min \{1,\|\boldsymbol{y}\| /\|\boldsymbol{x}-\boldsymbol{y}\|\}$, one easy checks that $\left|t-x^{0}\right| \leqq\|\boldsymbol{x}\|$, and $\left|t-y^{0}\right| \leqq\|\boldsymbol{y}\|$, and so the lemma holds.

In what follows we shall use the following property $(\mathrm{p})$ of the real line:

(p) Let $I_{i}$ be a family of bounded, closed intervals. If the intervals $I_{i}$ are pairwise intersecting, then the intersection of the whole family is non-empty.

It is to be noted that if $A \subset M$, and $l \cap A=\emptyset$, then $t \in \cap\{I(l ; x): x \in A\}$ if and only if $a+t v \in A^{\perp}$.

Proposition 1. Let $A \subset M$, and let $D$ be a maximal $\perp$-set in $A^{\perp \perp}$. If $\operatorname{l\cap } A^{\perp}=\emptyset$, then $l \cap D \neq \emptyset$.

Proof. First of all let us show that for every $x, x^{\prime} \in A^{\perp}, I(l ; x) \cap I\left(l ; x^{\prime}\right) \neq \emptyset$. Consider

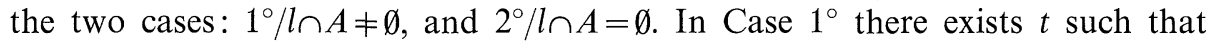
$a+t v \in A$. Since $a+t v \perp x$, and $a+t v \perp x^{\prime}$, it follows that $t \in I(l ; x) \cap I\left(l ; x^{\prime}\right)$. In Case $2^{\circ}$, since $\ln A=\emptyset$, and $\ln A^{\perp}=\emptyset$, it follows by (p) that there exist $x_{1}, x_{2} \in A$ such that $I\left(l ; x_{1}\right) \cap I\left(l ; x_{2}=\emptyset\right.$. Let $I\left(l ; x_{i}\right)=\left[a_{i}, b_{i}\right], b_{1}<a_{2}$. Now, since $x \perp x_{i}, I(l ; x) \cap I\left(l ; x_{i}\right) \neq \emptyset$ and so, $\left[b_{1}, a_{2}\right] \subset I(l ; x)$. In the same way $\left[b_{1}, a_{2}\right] \subset I\left(l ; x^{\prime}\right)$. Therefore in either case $I(l ; x) \cap I\left(l ; x^{\prime}\right) \neq \emptyset$.

It follows now, by Lemma 1 and $(\mathrm{p})$, that $\cap\left\{I(l ; x): x \in D \cup A^{\perp}\right\} \neq \emptyset$. Thus, there exists $t$ such that $a+t v-x \notin C$ for $x \in D$ and $x \in A^{\perp}$. Since $a+t v \perp A^{\perp}$, it follows that $a+t v \in A^{\perp \perp}$, and would $l \cap D=\emptyset$, then $a+t v \in D^{\perp}$, what would contradict to the assumed maximality of $D$ in $A^{\perp \perp}$. Therefore $a+t v \in D$. Q.E.D.

Corollary 1. If $E$ is a maximal $\perp$-set in $M$, then $l \cap E \neq \emptyset$, and $s o,(M, \perp)$ is a $D$-space.

Proof. Set $A=M$ in Proposition 1. It follows that $l \cap E \neq \emptyset$. Let $x \neq y, x, \not y$ be two points of $M$, and let $E$ be a maximal $\perp$-set. Define $l=\{x+t(y-x): t \in R\}$. Since $l \cap E \neq \emptyset$, there exists $z \in E \cap l$. But then $z \not \subset x$, and $z \not \subset y$, since $\{x, y, z\} \subset l$. So the condition (D) of Section 2 holds. Q.E.D.

Theorem 1. $(M, \perp)$ is a complete Dacey space and so, $L(M, \perp)$ is a complete orthomodular lattice.

Proof. Let $A=A^{\perp \perp} C M$, and let $D$ be a maximal $\perp$-set in $A$. To show that $D^{\perp \perp}=A$ it is enough to show that $D^{\perp} \subset A^{\perp}$. Suppose, to the contrary, that $x \in D^{\perp}$ and $x \notin A^{\perp}$. Since $D$ is a maximal $\perp$-set in $A, x \notin A$. Therefore there exists $y \in A, y \neq x$ such that $y \not \subset x$. Let $l=\{x+t(y-x): t \in R\}$. Since $l \cap A \neq \emptyset$ it follows that $l \cap A^{\perp}=\emptyset$ and so, by Proposition $1, l \cap D \neq \emptyset$. This, however, contradicts to $x \in l \cap D^{\perp}$. Q.E.D. 


\section{Final Remarks}

A natural topology of $M$ is given by a norm $\|x\|_{a, v}=\left|x^{0}\right|+\|\boldsymbol{x}\|$. The topology is independent of the choice of $(a, v) \in M \times C$, and so, it generates a natural Borel structure. Observe that a maximal $\perp$-set is automatically closed, and so is a Borel set. Morever, if $A \subset M$ is Borel, then $A^{\perp}$ is also a Borel set. It follows that the subset $L^{B}(M, \perp)$ of $L(M, \perp)$ consisting of all Borel sets in $L(M, \perp)$ is a $\sigma$-complete, orthomodular lattice.

Lemma 1. If $A=A^{\perp \perp}$ is Borel, and $D$ is a maximal $\perp$-set in $A$, then $D$ is also Borel.

Proof. Let $D^{\prime}$ be a maximal $\perp$-set in $A^{\perp}$, and let $E=D \cup D^{\prime}$. Then $E$ is a maximal $\perp$-set, and so $E$ is Borel. But $D=E-A^{\perp}$, therefore $D$ is also Borel. Q.E.D.

It follows that $A \in L^{B}(M, \perp)$ if and only if $A=D^{\perp \perp}, D$ being a Borel $\perp$-set.

Having in mind a possible need for description of "faster than light" particles, it is interesting to consider the anticausal logic $L(M, T)$, where " $T$ " means "time- or light-like". In this case one easily proves that $(M, T)$ is a $D$-space but, if $\operatorname{dim} V>2$, is not a (CD)-space. Therefore $L(M, T)$ is an ortho-modular orthoposet but not even a lattice.

Finally, observe that every automorphism of the causal logic $L(M, \perp)$ is induced by a transformation of $M$ preserving interiors of light cones, and so, by the result of Borchers and Hegerfeldt [6], is a Poincaré transformation or dilation.

\section{References}

1. Domotor, Z. : Synthese 24, 5 (1972)

2. Zeeman, E.C.: J. Math. Phys. 5, 490 (1964)

3. Alonso,J.L., Yndurain, F. Y.: Commun. math. Phys. 4, 349 (1967)

4. Teppati,G.: Nuovo Cimento 54 A, 800 (1968)

5. Gheorghe,C., Mihul,E.: Commun. math. Phys. 14, 165 (1969)

6. Borchers, H.J., Hegerfeldt,G.C.: Commun. math. Phys. 28, 259 (1972)

7. Cegła,W., Jadczyk, A.Z.: Rep. Math. Phys. 9, 377 (1976)

8. Randall,C.H., Foulis, D. J.: The empirical logic approach to the physical sciences. In : Lecture notes in physics, Vol. 29. Foundations of quantum mechanics and ordered linear spaces. BerlinHeidelberg-New York: Springer 1972

9. Dacey,J.C.: Caribbean J. Sci. Math. 1, 51 (1969)

10. Foulis, D.J., Randall,C.H.: J. Math. Phys. 13, 1667 (1972)

11. Foulis, D.J., Randall, C.H. : J. Combinatorial Theory 11, 157 (1971)

12. Woo, Ching-Hung: Quantum effects on the accuracy of momentum measurements. Preprint, Inst. Adv. Study. Princeton 1964

13. Dollard,J.D.: Commun. math. Phys. 12, 193 (1969)

14. Jauch,J.M., Lavine, R., Newton, R.G.: Helv. Phys. Acta. 45, 325 (1972)

15. Finkelstein, D. : Phys. Rev. D9, 2219 (1974)

Communicated by R. Haag 
